\section{Biomechanical optimal control of human arm motion}

\author{
R Maas and S Leyendecker
}

Proc IMechE Part K:

Multi-body Dynamics

227(4) 375-389

(C) IMechE 2013

Reprints and permissions:

sagepub.co.uk/journalsPermissions.nav DOI: 10.1 |77//4644193|3488363

pik.sagepub.com

(3)SAGE

\begin{abstract}
As both ordinary and well-trained human motion is mostly planned and controlled unconsciously by the central nervous system (CNS), human control mechanisms remain relatively obscure. Despite, they are an interesting topic, for example, with regard to improve protheses or athletic motion. To learn and understand more about the control of human motion, we use rigid multibody systems to represent bones and joints and formulate an optimal control problem (OCP) with the goal to minimise a physiologically motivated cost function, while the equations of motion and further nonlinear constraints have to be fulfilled. The investigated biomechanical movements are induced either via joint torques or via Hilltype muscle forces. We compare several cost functions known from literature to another one concerning the impact on the joints by involving the constraint forces. A direct transcription method called DMOCC (discrete mechanics and optimal control for constraint systems) is used to solve the OCP, whereby we benefit from its structure preserving formulation, as the resulting optimal discrete trajectories are symplectic-momentum preserving.
\end{abstract}

\title{
Keywords
}

Biomechanics, optimal control, multibody dynamics, Hill-type muscle model, DMOCC, structure preserving integration

Date received: 31 January 20I3; accepted: 5 April 2013

\section{Introduction}

Besides a variety of other physics-based approaches to simulate human motion, optimal control of biomechanical motion is a topic of growing interest (see Ref. 1

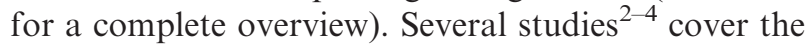
question, how such motion is controlled by the central nervous system (CNS) in the context of walking and running simulations. Most of these studies aim for applying the results to optimally controlled robots or for improving the design of protheses. Further on, multiple studies are engaged in optimal control of human arm or finger motion and investigate different optimality criteria or cost functions. A criterion of minimum torque change is favoured in Ref. 5, whereas Refs. 6 and 7 introduce a criterion of minimum jerk during the motion. Accounting for the comfort during a motion, a criterion is developed, in Refs. 8 and 9, saying that the motion is constantly compared to known postures and the discomfort of the motion is quantified via the difference of the joint angles to known comfort positions.

The present work investigates the optimal control of human arm motion in a similar way, comparing different optimal control criteria and cost functions to understand which criterion leads to the most realistic motion, i.e. which criterion resembles the control of the human CNS most closely. We investigate several well-known physiologically motivated cost functions and criteria similar to those in Refs. 5-7 and illustrate an idea to formulate a criterion that reduces the impact on the joints during a motion. Herein, it is of particular importance that we are solving an index 3 system. Therewith the constraint forces can be directly accessed and used within a cost function of the optimal control problem (OCP). This criterion could be of interest for the ergonomic design of everyday or industrial processes. In a more medical context, it could help to explain pathologic motion and confirm a diagnosis, e.g. whether a patient is using relieving postures to minimise pain or discomfort.

Chair of Applied Dynamics, University of Erlangen-Nuremberg, Erlangen, Germany

\section{Corresponding author:}

R Maas, Chair of Applied Dynamics, University of Erlangen-Nuremberg, Konrad-Zuse-Str. 3/5, 91052 Erlangen, Germany.

Email: ramona.maas@ltd.uni-erlangen.de 
To model the human arm, we use a multibody dynamics formulation as explained, for example, in Refs. 10-12 and add a formulation of a cardan joint for modelling the two degrees of freedom in both the elbow and the wrist, respectively. What clearly distinguishes our work from other related studies is that the optimal control problem is solved using DMOCC (discrete mechanics and optimal control for constraint systems ${ }^{10}$ ), which implicates the benefit of structure preserving behaviour of the approximate solution. This means that the resulting optimal discrete trajectories exactly represent certain characteristics of the real system like for example consistency of momentum maps and symplecticity. It is commonly known that using typical commercial available integrators like for example Matlab's ODE solvers, which rely on discretising the continuous equations of motion, may lead to numerical dissipation or artificial gain in energy or angular momentum during a dynamical simulation. Accordingly, the applied forces and torques are not exactly translated into motion of the system. However, during optimal control simulations, it is crucial that angular momentum is represented correctly. This means, if no gravity or actuation is present and a system moves due to initial velocities, then the angular momentum is conserved; if forces or torques actuate the system, then the angular momentum changes only and exactly according to the applied forces and torques. Any artificial dissipation or gain of energy or angular momentum influences both the convergence behaviour and the reliability of the results of an optimal control simulation. As this effect increases with the size of the time step, one possibility to improve angular momentum representation in standard methods is to use adequate time step sizes, with the disadvantage of a larger number of optimisation variables. And even if the numerical dissipation or gain gets smaller with decreasing time steps, it will not be zero to numerical accuracy. In contrast, a variational integrator as for example explained in Refs. 10 and 12 yields exact representation of angular momentum and a good energy behaviour, even for long-term dynamical simulations with large time steps (for example, see Ref. 12 or Ref. 13 including muscle actuation) and for optimal control simulations with challenging nonlinear constraints, see Refs. 10 and 14 . This work is mainly based on the approach discussed in Ref. 14.

As a further step of the work, the joint torque actuation of the elbow is replaced by Hill-type muscle models of the seven most important muscles around the human elbow. The selection of muscles and their parameters are adapted from Refs. 15 and 16, while taking into account our model geometry. Similar to an approach by Ref. 17, we suppose the muscle path is always the shortest possible connection between two insertion points, as muscles are known to have a tone even at rest. ${ }^{18}$ This approach yields muscle paths that wrap around bodies and joints and lead to more realistic force directions and length evolutions of the muscles, in contrast to typical approaches, ${ }^{15,16,19,20}$ where muscle connections are either defined by straight lines or connections around user defined via points. Our new contribution is to use such dynamical changing muscle paths within an optimal control simulation. As the muscle force depends on the length, velocity and the direction of the muscle path, we suppose this is of high importance to get reasonable results from optimal control simulations.

In the following section, a structure preserving discrete formulation for constrained forced multibody dynamics is briefly explained. A more detailed description on this topic can be found in Refs. 10 12 and 21. Then, this formulation is augmented by the implementation of muscle actuation with Hill-type muscle models containing the typical force-length and force-velocity behaviour as well as a parallel elastic component. Further on, the optimal control problem is formulated, together with its constraints. A way to determine the constraint forces and torques, when using the introduced rigid body formulation, is shown for single kinematic pairs connected with a spherical or a cardan joint. Finally, a kinematic chain is set up consisting of these joints, which is further on used to model a human arm. In the last section, we compare all introduced cost functions and the optimal motions they yield, using an example of a human arm performing a steering motion and an example of lifting the arm with muscle actuation in the elbow.

\section{Forced constrained rigid body dynamics}

The configuration of a rigid body in space, as illustrated on the left side of Figure 1, can be characterised by a vector $\boldsymbol{q} \in \mathbb{R}^{12}$ of redundant coordinates, including the location of the centre of mass $\boldsymbol{\varphi} \in \mathbb{R}^{3}$ and the orthonormal body frame $\boldsymbol{R}=\left[\begin{array}{lll}\boldsymbol{d}_{1} & \boldsymbol{d}_{2} & \boldsymbol{d}_{3}\end{array}\right] \in S O(3)$ located in the centre of mass, e.g. $\boldsymbol{d}_{i}, i=1,2,3$ aligned with the principal axes of inertia

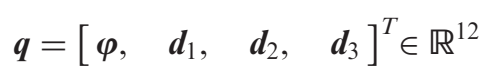

Connecting several rigid bodies via joints leads to kinematic chains. The smallest kinematic chain is a pair of rigid bodies as depicted in the middle part of Figure 1. The configuration variable of a kinematic chain with $K$ bodies consists of $\boldsymbol{q}^{\alpha}, \alpha=1, \ldots, K$ in the form of equation (1). This yields a $k$-dimensional configuration variable with $k=12 \mathrm{~K}$

$$
\boldsymbol{q}=\left[\boldsymbol{q}^{1}, \ldots, \boldsymbol{q}^{K}\right]^{T} \in \mathbb{R}^{k}
$$

Locations $\rho^{\alpha}$ on the $\alpha$-th body, for instance the location of joints connecting two bodies or the 
muscle insertion points, can be described via coordinates $\rho_{i}^{\alpha}, i=1,2,3$ in the respective body frame

$$
\boldsymbol{\rho}^{\alpha}=\boldsymbol{R}^{\alpha} \cdot\left[\rho_{1}^{\alpha}, \rho_{2}^{\alpha}, \rho_{3}^{\alpha}\right]^{T}
$$

\section{Constrained multibody system}

In addition to $m_{\text {int }}=6$ internal constraints $\boldsymbol{g}_{\text {int }}(\boldsymbol{q})=\mathbf{0}$ that account for the body frame remaining orthonormal, coupling neighbouring bodies via joints leads to $m_{\text {ext }}^{J}$ external constraints $\boldsymbol{g}_{\text {ext }}^{J}(\boldsymbol{q})=\boldsymbol{0}$ on the system, depending on the joint type. The external constraints reduce the degrees of freedom of the relative motion of one body with respect to the other from 6 to $r^{J}=6-m_{\text {ext }}^{J}$. The Jacobian of the external constraints is further on denoted by $\boldsymbol{G}_{\text {ext }}^{J}(\boldsymbol{q})=D \boldsymbol{g}_{\text {ext }}^{J}(\boldsymbol{q}) \in R^{m_{\text {ext }} \times k}$. Altogether, this yields $m=m_{\text {int }}+m_{\text {ext }}^{J}$ constraints on the system $\boldsymbol{g}^{J}(\boldsymbol{q})=\left[\boldsymbol{g}_{\text {int }}(\boldsymbol{q}), \boldsymbol{g}_{\text {ext }}^{J}(\boldsymbol{q})\right]^{T}$.

\section{Actuation of a multibody system}

A rigid multibody system can be actuated via a vector of joint torques $\tau^{J} \in \mathbb{R}^{r^{J}}$, whose dimension depends on the number of degrees of freedom $r^{J}$ of the specified joint connection. In addition, an actuation via forces and torques directly on the rigid bodies $\tau_{r b} \in \mathbb{R}^{6}$ is possible.

Combined, the generalised forces actuating a $k$ dimensional kinematic chain consisting of $K$ bodies connected via $K-1$ joints read

$$
\left[\tau_{r b}, \tau^{J_{1}}, \ldots, \tau^{J_{K-1}}\right]^{T} \in \mathbb{R}\left(6+\sum_{i=1}^{K-1} r^{J_{i}}\right)
$$

The redundant forces on each body $\boldsymbol{f}=\left[\boldsymbol{f}^{1}, \ldots, \boldsymbol{f}^{K}\right]^{T} \in \mathbb{R}^{k}$ can be calculated from

$$
\boldsymbol{f}=\boldsymbol{B}(\boldsymbol{q})^{T} \cdot\left[\boldsymbol{\tau}_{r b}, \tau^{J_{1}}, \ldots, \tau^{J_{K-1}}\right]^{T}
$$$$
\text { with } \boldsymbol{B}(\boldsymbol{q}) \in \mathbb{R}^{k \times\left(6+\sum_{i=1}^{K-1} r^{J_{i}}\right)} \text { representing the configura- }
$$
tion-dependent input transformation matrix in the form of equation (42) in Ref. 10. Instead of using joint torques, the actuation on each body can be represented equivalently by forces $\boldsymbol{h}_{\varphi} \in \mathbb{R}^{3}$ and torques $\boldsymbol{h}_{\theta} \in \mathbb{R}^{3}$ in the centre of mass of each body $\boldsymbol{h}=\left[\boldsymbol{h}_{\varphi}^{1}, \boldsymbol{h}_{\theta}^{1}, \ldots, \boldsymbol{h}_{\varphi}^{K}, \boldsymbol{h}_{\theta}^{K}\right]^{T} \in \mathbb{R}^{6 K}$, see right part of Figure 1, via

$$
\boldsymbol{h}=\boldsymbol{P}_{i n t}^{T}(\boldsymbol{q}) \cdot \boldsymbol{f}
$$

with $\boldsymbol{P}_{\text {int }}(\boldsymbol{q}) \in \mathbb{R}^{k \times(k-m)}$ representing the internal nullspace matrix, exemplarily specified later in equation (17) for a pair (see Ref. 11 for details).

\section{Nonlinear Hill-type muscle actuation}

Let the system be actuated by a Hill-type muscle force, ${ }^{22}$ see Figure 2, then the scalar force amount of the muscle force $F_{n}^{M}$ acting during a time interval $\left[t_{n}, t_{n+1}\right]$ can be calculated via

$$
F_{n}^{M}=F_{n}^{C C}+F_{n}^{P E C}
$$

Assuming the parallel elasticity of the muscle to be proportional to the length of the muscle element $l^{M}$ with the proportionality constant $k_{p}$ yields

$$
F_{n}^{M}=f_{l}\left(l_{n}^{M}\right) f_{v}\left(v_{n}^{M}\right) A_{n} F_{\max }+k_{p} l_{n}^{M}
$$

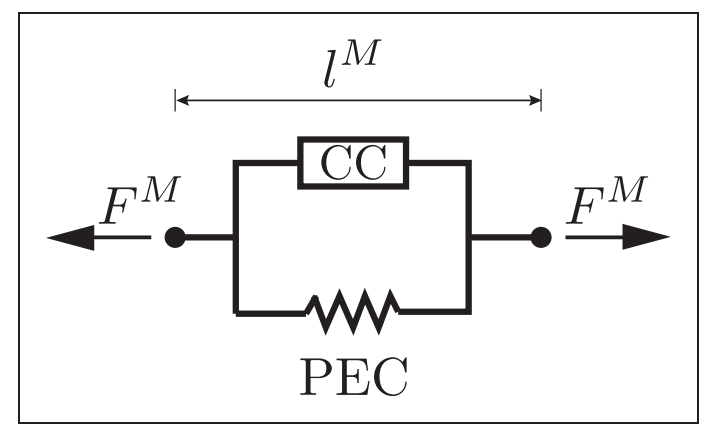

Figure 2. Hill-type muscle model.

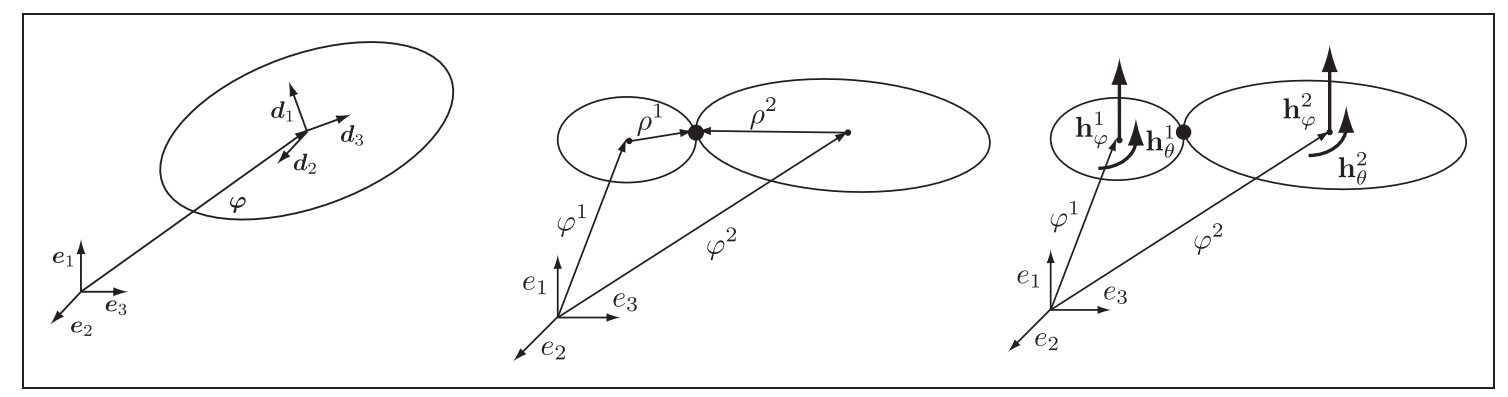

Figure I. Configuration of a rigid body in space (left) and a pair of rigid bodies connected by a joint (middle), subject to forces and torques in the centre of mass (right). 
Herein, $f_{l}\left(l^{M}\right) \in[0,1]$ is a function representing the force-length relation of the muscle, $A \in[0,1]$ is the activity of the muscle and $F_{\max }$ is the maximal possible muscle force. The typical Hill-hyperbola forcevelocity relation is given by the function $f_{v}\left(v^{M}\right) \in[0,1.4]$ with the contraction velocity of the muscle approximated via $v_{n}^{M}=\frac{l_{n+1}^{M}-l_{n}^{M}}{\Delta t}$ and the time step length denoted by $\Delta t$. The muscle force $\tau_{n}^{M}$ acting on the body is given by a multiplicative set up of the scalar force value and the unit vector $\boldsymbol{r}_{n}$ of the force direction

$$
\tau_{n}^{M}=F_{n}^{M} \boldsymbol{r}_{n}
$$

Force-length behaviour. In equation (3), the force-length relation of the muscle model is described via the function $f_{l}\left(l_{n}^{M}\right)$, given by

$f_{l}\left(l^{M}\right)=\left\{\begin{array}{cl}\left(\frac{-1}{\left(l_{o p t}-l_{1}\right)^{2}\left(l^{M}-l_{o p t}\right)^{2}+1}\right) & \text { if } l_{1}<l^{M}<2 l_{o p t}-l_{1} \\ 0 & \text { else }\end{array}\right.$

where $l_{\text {opt }}$ is the optimal muscle length yielding $f_{l}=1$. By adapting the muscle parameters $l_{\text {opt }}$ and $l_{1}$ to the geometry of our model, it is ensured that $f_{l}$ is mostly in the parabolic part.

Force-velocity behaviour. The force-velocity behaviour of a muscle is modelled with the typical Hillhyperbolic relation

$$
f_{v}\left(v^{M}\right)=\left\{\begin{array}{lll}
\frac{v_{\max }-v^{M}}{v_{\max }+\frac{v^{M}}{\gamma}} & \text { if } & v^{M}>0 \\
\frac{B_{1}}{\frac{v^{M}}{v_{\max }}-B_{2}}+B_{3} & \text { if } & v^{M}<=0
\end{array}\right.
$$

containing the maximal possible contraction velocity of a muscle $v_{\max }$, which has to be determined experimentally. For the lack of experimental data here, we use a value of $v_{\max }$ being $2 l_{\text {opt }}$ per second to keep the $f_{v}$ value in a realistic range during our simulations. The curvature of the concentric part is described with the muscle-related constant parameter $\gamma \in \mathbb{R}$ adapted from Refs. 13 and 23, whereas the eccentric part is described by a mirrored hyperbola, with experimentally determined constant parameters $B_{1}, B_{2}, B_{3} \in \mathbb{R}$ (see Ref. 24).

This means, to calculate the actual muscle force, we need the actual muscle length in every time step as well as the force directions at the insertion points of the muscle. The muscle length and force direction is particularly related to the joint angle. Several studies use an alterable number of artificial 'via' points or 'wrapping' points to relate the muscle path to the joint angle, see for example Ref. 15. The determination of such artificial points requires a lot of anatomical knowledge, which is not yet available for all biomechanical structures and the results are quite sensitive to the location of such points. In software packages like OpenSim, so-called muscle moment arms are then calculated as partial derivatives of the muscle length around those artificial points with respect to the joint angle, see for example Refs. 15 and 25.

Our approach is to assume that the muscles and tendons are always under tension as it is described for example in Ref. 18, which means that tendons and muscles are supposed to follow the path of minimal distance between two insertion points, around the bodies. Within our multibody simulation framework for biomechanical systems, we represent bones and joints via mostly smooth bodies like cylinders and spheres. It is known that the shortest path on the surface of a cylinder is a helix and the shortest path on a sphere is an orthodrome. We want to reduce computational effort in finding the path of minimal length around bodies and joints with an algorithm that determines this path as a G1-continuous combination of straight lines (wherever possible, i.e. whenever the straight line does not intersect the bodies or joints), helices and orthodromes. Note that G1 (geometrical) continuously joined curves share tangential directions, while the length of the tangent vectors might differ. Thus, the length of this path can directly be calculated as the sum of the length of the single parts and the force direction is given via the tangent vector at the insertion points. In Figure 3, an exemplarily muscle path resulting from this semi-analytical calculation is shown, which is in this case a G1-continuous helix-line-helix combination.

\section{Structure preserving discrete equations of motion}

A common way to describe the evolution of timecontinuous mechanical systems is given by Lagrangian mechanics. In the context of variational integrators (see Ref. 12) instead of the discretisation of the continuous equations of motion, a time stepping scheme is derived via a discrete variational principle. As a consequence, it inherits the structure preserving characteristics of the real system. ${ }^{21}$ Basically this means that the discrete system exactly represents the behaviour of the analytical system concerning the consistency of momentum maps or symplecticity. Further on, the results show a good energy

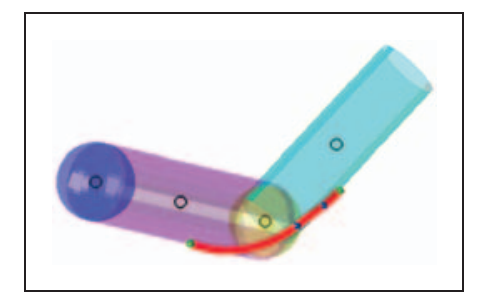

Figure 3. Example of a helix-line-helix muscle path calculated with the semi-analytical procedure. 
behaviour in the sense that no artificial loss or gain in total energy of the system can be noticed even for long simulation periods.

Consider a $k$-dimensional forced mechanical system discretised with a constant time step $\Delta t \in \mathbb{R}$, then the configuration variable $\boldsymbol{q}:\left[t_{0}, t_{N}\right] \rightarrow \mathbb{R}^{k}$ at $t_{n}=t_{0}+n \Delta t$ is approximated via $\boldsymbol{q}_{d}:\left\{t_{0}, t_{0}+\right.$ $\left.\Delta t, \ldots, t_{0}+N \Delta t=t_{N}\right\} \rightarrow \mathbb{R}^{k}, N \in \mathbb{N} \quad$ with $\quad \boldsymbol{q}_{n}=$ $\boldsymbol{q}_{d}\left(t_{n}\right)$. Let the system be constrained by $m$ constraints resulting from the rigid body formulation and the joints in between the bodies. For the Jacobian of the constraints, $\boldsymbol{G}_{d}\left(\boldsymbol{q}_{n}\right)=\Delta t \boldsymbol{G}\left(\boldsymbol{q}_{n}\right)$ is used in the discrete case, while the discrete Lagrangian $L_{d}: \mathbb{R}^{k} \times R^{k} \rightarrow \mathbb{R}$ approximates the action during one time step $\Delta t$. Further on, the system is actuated by the discrete generalised forces $\tau_{d}=\left\{\tau_{n}\right\}_{n=0}^{N-1}$. With these approximations and $D_{1}, D_{2}$ indicating the differential operators with respect to the first and second argument of $L_{d}$, the discrete equations of motion of the problem are given by

$$
\begin{aligned}
& \boldsymbol{P}^{T}\left(\boldsymbol{q}_{n}\right) \cdot\left[D_{2} L_{d}\left(\boldsymbol{q}_{n-1}, \boldsymbol{q}_{n}\right)\right. \\
& \left.\quad+D_{1} L_{d}\left(\boldsymbol{q}_{n}, \boldsymbol{F}\left(\boldsymbol{u}_{n+1}, \boldsymbol{q}_{n}\right)\right)+\boldsymbol{f}_{n-1}^{+}+\boldsymbol{f}_{n}^{-}\right]=\mathbf{0}
\end{aligned}
$$

where $\boldsymbol{P}\left(\boldsymbol{q}_{n}\right) \in \mathbb{R}^{k \times(k-m)}$ denotes the discrete nullspace matrix (for further details see Ref. 11). The discrete force $\boldsymbol{f}_{n-1}^{+}$denotes the effect of the generalised force $\tau_{n-1}$ acting in the time interval $\left[t_{n-1}, t_{n}\right]$ on $\boldsymbol{q}_{n}$, while $\boldsymbol{f}_{n}^{-}$ is coming from the effect of $\tau_{n}$ acting in $\left[t_{n}, t_{n+1}\right]$ on $\boldsymbol{q}_{n}$.

For muscle forces, these discrete forces can be calculated from

$$
\boldsymbol{f}_{n-1}^{+}=\frac{h}{2} \boldsymbol{B}^{T}\left(\boldsymbol{q}_{n}\right) \cdot \tau_{n-1}^{m}=\frac{h}{2} \boldsymbol{B}^{T}\left(\boldsymbol{q}_{n}\right) \cdot F_{n-1}^{M}\left(\boldsymbol{q}_{n-1}, \boldsymbol{q}_{n}\right) \boldsymbol{r}_{n-1}
$$

$$
\boldsymbol{f}_{n}^{\llcorner}=\frac{h}{2} \boldsymbol{B}^{T}\left(\boldsymbol{q}_{n}\right) \cdot \tau_{n}^{m}=\frac{h}{2} \boldsymbol{B}^{T}\left(\boldsymbol{q}_{n}\right) \cdot F_{n}^{M}\left(\boldsymbol{q}_{n}, \boldsymbol{q}_{n+1}\right) \boldsymbol{r}_{n}
$$

Herein, the matrix $\boldsymbol{B}\left(\boldsymbol{q}_{n}\right)$ contains the lever arms and assigns the forces to the bodies.

Remark. Due to the usage of a variational integrator, we can guarantee that angular momentum is represented correctly. This means, if the sum of actuating forces vanishes, we have exact conservation of linear momentum, if the resulting torques sum up to zero, the angular momentum is exactly conserved. In the case of muscle actuation, force directions and lever arms of one muscle being attached at different bodies are typically not equal, i.e. the resulting forces and torques do not sum up to zero, yielding a change in angular momentum, which is represented consistently according to the actuating forces and torques, if a structure preserving integrator is used. Due to the premultiplication of the equations with the matrix $\boldsymbol{P}\left(\boldsymbol{q}_{n}\right)$ having the property range $\left(\boldsymbol{P}\left(\boldsymbol{q}_{n}\right)\right)=\operatorname{null}\left(\boldsymbol{G}_{d}\left(\boldsymbol{q}_{n}\right)\right)$, the discrete constraint forces $\boldsymbol{G}_{d}^{T}\left(\boldsymbol{q}_{n}\right) \cdot \lambda_{n}$ and therefore the calculation of the Lagrange multipliers $\lambda_{n} \in \mathbb{R}^{m}$ and the related conditioning issues are avoided. Due to the usage of the discrete nullspace method and a discrete reparametrisation $\boldsymbol{q}_{n+1}=\boldsymbol{F}\left(\boldsymbol{u}_{n+1}, \boldsymbol{q}_{n}\right)$ with the discrete generalised coordinates $\boldsymbol{u}_{d}=\left\{\boldsymbol{u}_{n}\right\}_{n=0}^{N}$ and $\boldsymbol{g}\left(\boldsymbol{q}_{n+1}\right)=\boldsymbol{g}\left(\boldsymbol{F}\left(\boldsymbol{u}_{n+1}, \boldsymbol{q}_{n}\right)\right)$ $=0$ fulfilling the constraints, the dimension of the system (4) is the minimum possible dimension $(k-m)$.

\section{The optimal control problem}

For multibody systems connected via joints, there exists an infinite number of possible trajectories and force fields to perform a motion from a predefined start configuration $\boldsymbol{q}\left(t_{0}\right)=\boldsymbol{q}_{0}$ and conjugate momentum $\boldsymbol{p}\left(t_{0}\right)=\boldsymbol{p}_{0}$ to a given end configuration $\boldsymbol{q}\left(t_{N}\right)=\boldsymbol{q}_{N}$ and conjugate momentum $\boldsymbol{p}\left(t_{N}\right)=\boldsymbol{p}_{N}$. Our goal is to formulate and solve an optimal control problem as illustrated in equation (7) and to use an appropriate discrete objective $J_{d}$, or respectively a discrete cost function $C_{d}$, to find a trajectory and force field that minimise this objective. At the same time, the discrete equations of motion (4) and boundary conditions (for example, to perform a rest-to-rest manoeuvre $\left.\boldsymbol{p}_{0}=\boldsymbol{p}_{N}=\boldsymbol{0}\right)$ must be fulfilled. There may be further constraints like bounds on the optimisation variables and path constraints (equality or inequality), e.g. to set limits on the joint angles according to anatomical restrictions

$$
\begin{aligned}
\min _{\boldsymbol{u}_{d}, \boldsymbol{\tau}_{d}} J_{d}\left(\boldsymbol{u}_{d}, \tau_{d}\right)= & \min _{\boldsymbol{u}_{d}, \boldsymbol{\tau}_{d}} \sum_{n=0}^{N-1} C_{d}\left(\boldsymbol{u}_{n}, \boldsymbol{u}_{n+1}, \tau_{n}\right) \\
\text { subject to : } & \text { fulfilment of the discrete } \\
& \text { equations of motion (4) } \\
& \cdot \text { initial and final conditions } \\
& \cdot \text { path constraints }
\end{aligned}
$$

\section{Optimal control simulations with joint actuation}

For simulations with joint actuation, we use several discrete cost functions and compare the resulting trajectories. A typical and in the field of robotic and automation widely used criterion for optimal motion is the minimisation of control effort

$$
J_{d}\left(\boldsymbol{u}_{d}, \tau_{d}\right)=\frac{1}{2} \Delta t \sum_{n=1}^{N-1}\left(\tau_{n}\right)^{T} \cdot \tau_{n}
$$

A further criterion introduced in Ref. 5 accounts for the minimisation of the change in torques during the simulation

$$
J_{d}\left(\boldsymbol{u}_{d}, \tau_{d}\right)=\frac{1}{2} \Delta t \sum_{n=1}^{N-2}\left(\frac{\tau_{n+1}-\tau_{n}}{\Delta t}\right)^{T} \cdot\left(\frac{\tau_{n+1}-\tau_{n}}{\Delta t}\right)
$$


The third goal is to perform the motion with minimal kinetic energy (similar to Ref. 7)

$$
J_{d}\left(\boldsymbol{u}_{d}, \boldsymbol{\tau}_{d}\right)=\frac{1}{2} \Delta t \sum_{n=1}^{N-1}\left({ }^{Q} \boldsymbol{p}_{n}^{+}\right)^{T} \cdot \boldsymbol{M}^{-1} \cdot{ }^{Q} \boldsymbol{p}_{n}^{+}
$$

with the constant symmetric positive definite mass matrix $\boldsymbol{M} \in \mathbb{R}^{k \times k}$ and the conjugate momentum

$$
{ }^{Q} \boldsymbol{p}_{n}^{+}=\boldsymbol{Q}\left(\boldsymbol{q}_{n}\right) \cdot\left[D_{2} L_{d}\left(\boldsymbol{q}_{n-1}, \boldsymbol{q}_{n}\right)+\boldsymbol{f}_{n-1}^{+}\right]
$$

where

$$
\boldsymbol{Q}\left(\boldsymbol{q}_{n}\right)=\boldsymbol{I}_{k \times k}-\boldsymbol{G}_{d}^{T} \cdot\left[\boldsymbol{G}_{d} \cdot \boldsymbol{M}^{-1} \cdot \boldsymbol{G}_{d}^{T}\right]^{-1} \cdot \boldsymbol{G}_{d} \cdot \boldsymbol{M}^{-1}
$$

This projected momentum fulfils the hidden constraints on momentum level and is explained in detail in Refs. 10 and 12. Finally, we want to compare these commonly known cost functions to a cost function that takes into account the impact on the joints. We therefore use a formulation that represents an approximation of the integral over the sum of squares of all constraint forces $\boldsymbol{F}_{\varphi}$ and torques $\boldsymbol{T}_{\theta}$ in the joints

$$
J_{d}\left(\boldsymbol{u}_{d}, \boldsymbol{\tau}_{d}\right)=\frac{1}{2} \Delta t \sum_{n=1}^{N-1}\left[\left(\boldsymbol{F}_{\varphi}\right)_{n}^{T} \cdot\left(\boldsymbol{F}_{\varphi}\right)_{n}+\left(\boldsymbol{T}_{\theta}\right)_{n}^{T} \cdot\left(\boldsymbol{T}_{\theta}\right)_{n}\right]
$$

In the next chapter, we show a possibility to determine the constraint forces using the introduced rigid body formulation for several pairs and a kinematic chain representing a human arm.

\section{Optimal control simulations with muscle actuation}

The implementation of muscle actuation yields the possibility to relate the optimality criteria to muscle behaviour. Within such simulations, we use for example criteria that involve the activity of the muscles. The first goal is to minimise the total amount of activities, together with the joint torques in shoulder and wrist

$$
\begin{aligned}
& J_{d}\left(\boldsymbol{u}_{d}, \tau_{d}^{J}, \boldsymbol{A}_{d}\right) \\
& \quad=\frac{1}{2} \Delta t \sum_{n=1}^{N-1}\left[\omega_{1}\left(\tau_{n}^{J}\right)^{T} \cdot \tau_{n}^{J}+\omega_{2}\left(\boldsymbol{A}_{n}\right)^{T} \cdot \boldsymbol{A}_{n}\right]
\end{aligned}
$$

Herein, the weighting factors $\omega_{1}, \omega_{2} \in \mathbb{R}$ ensure that the influence of torques and activities on the value of the cost function are in the same order of magnitude. Second, similar to the minimum control change criterion, see equation (9), we aim at minimising changes in activities and joint torques

$$
\begin{aligned}
& J_{d}\left(\boldsymbol{u}_{d}, \tau_{d}^{J}, \boldsymbol{A}_{d}\right) \\
& =\frac{1}{2} \Delta t \sum_{n=1}^{N-2}\left[\omega_{1}\left(\frac{\tau_{n+1}^{J}-\tau_{n}^{J}}{\Delta t}\right)^{T} \cdot\left(\frac{\tau_{n+1}^{J}-\tau_{n}^{J}}{\Delta t}\right)\right. \\
& \left.\quad+\omega_{2}\left(\frac{\boldsymbol{A}_{n+1}-\boldsymbol{A}_{n}}{\Delta t}\right)^{T} \cdot\left(\frac{\boldsymbol{A}_{n+1}-\boldsymbol{A}_{n}}{\Delta t}\right)\right]
\end{aligned}
$$

Further on, we use a criterion where the muscle force effort in the elbow is minimised (together with the joint torque effort in shoulder and wrist)

$$
\begin{aligned}
& J_{d}\left(\boldsymbol{u}_{d}, \tau_{d}^{J}, \boldsymbol{A}_{d}\right) \\
& \quad=\frac{1}{2} \Delta t \sum_{n=1}^{N-1}\left[\omega_{1}\left(\tau_{n}^{J}\right)^{T} \cdot \tau_{n}^{J}+\omega_{2}\left(\tau_{n}^{M}\right)^{T} \cdot \tau_{n}^{M}\right]
\end{aligned}
$$

Moreover, it is possible to weight the muscle forces in this cost function by the cross-sectional area of the muscles, like it is done in Ref. 26, to evaluate muscle stresses. This criterion demands larger force amounts from thicker muscles, which can recover better than thinner ones. Hence, this criterion is supposed to lead to motions that can be performed with maximum endurance

$$
\begin{aligned}
& J_{d}\left(\boldsymbol{u}_{d}, \tau_{d}^{J}, \boldsymbol{A}_{d}\right) \\
& \quad=\frac{1}{2} \Delta t \sum_{n=1}^{N-1}\left[\omega_{1}\left(\tau_{n}^{J}\right)^{T} \cdot \tau_{n}^{J}+\omega_{2}\left(\boldsymbol{\sigma}_{n}^{M}\right)^{T} \cdot \boldsymbol{\sigma}_{n}^{M}\right]
\end{aligned}
$$

with the muscle stress $\sigma_{i, n}=\frac{\tau_{i, n}^{M}}{P C S A_{i}}$ for the $i$ th muscle and its cross-sectional area $P S C A_{i}$, taken from Ref. 16. Within all of the above introduced musclerelated criteria, we have to find weighting factors $\omega_{1}, \omega_{2}$ to ensure that both parts of the cost function (muscle related and joint torque related) are minimised. In the second numerical example, we compare these more muscle-related criteria to the introduced criterion on minimising the constraint forces (11) which is supposed to yield an ergonomically optimal motion.

\section{Determination of constraint forces}

In order to prevent the dynamical system from violating the kinematic joint coupling constraints explained in section 'Constrained multibody system', constraint forces or - depending on the joint type - constraint torques are present in the joints. In the following, a formulation of two common joints and the computation of the related constraint forces are given. Further on, spherical and cardan joints are used to build the kinematic chain of the arm model. 


\section{Computation of the Lagrange multipliers}

Obviously the constraint forces and torques are directly related to the Lagrange multipliers. Due to the usage of a discrete nullspace method in equation (4), we save the calculation of the Lagrange multipliers in the first place to reduce computational effort and to exclude conditioning issues. However, it is possible to
Lagrange multipliers related to the constrained relative translation of the bodies, the external constraint forces are given by $\left(\boldsymbol{G}_{\text {ext }}^{S}(\boldsymbol{q})\right)^{T} \cdot \lambda_{\text {ext }}^{S}$. By premultiplying these redundant constraint forces with the transposed internal nullspace matrix $\boldsymbol{P}_{\text {int }}^{T}(\boldsymbol{q})$ following the formulation in equation (2), we finally get a set of constraint forces and torques acting in the centre of mass of the rigid bodies

$$
\left[\begin{array}{c}
\boldsymbol{h}_{\varphi}^{1} \\
\boldsymbol{h}_{\theta}^{1} \\
\boldsymbol{h}_{\varphi}^{2} \\
\boldsymbol{h}_{\theta}^{2}
\end{array}\right]=\boldsymbol{P}_{i n t}^{T} \cdot\left(\boldsymbol{G}_{\text {ext }}^{S}\right)^{T} \cdot \lambda_{\text {ext }}^{S}=\left[\begin{array}{cccccccc}
\boldsymbol{I} & 0 & 0 & 0 & 0 & 0 & 0 & 0 \\
0 & \widehat{\boldsymbol{d}_{1}^{1}} & \widehat{\boldsymbol{d}_{2}^{1}} & \widehat{\boldsymbol{d}_{3}^{1}} & 0 & 0 & 0 & 0 \\
0 & 0 & 0 & 0 & \boldsymbol{I} & 0 & 0 & 0 \\
0 & 0 & 0 & 0 & 0 & \widehat{\boldsymbol{d}_{1}^{2}} & \widehat{\boldsymbol{d}_{2}^{2}} & \widehat{\boldsymbol{d}_{3}^{2}}
\end{array}\right] \cdot\left[\begin{array}{c}
-\lambda_{\varphi} \\
-\rho_{1}^{1} \lambda_{\varphi} \\
-\rho_{2}^{1} \lambda_{\varphi} \\
-\rho_{3}^{1} \lambda_{\varphi} \\
\lambda_{\varphi} \\
\rho_{1}^{2} \lambda_{\varphi} \\
\rho_{2}^{2} \lambda_{\varphi} \\
\rho_{3}^{2} \lambda_{\varphi}
\end{array}\right]=\left[\begin{array}{c}
-\lambda_{\varphi} \\
-\rho^{1} \times \lambda_{\varphi} \\
\lambda_{\varphi} \\
\rho^{2} \times \lambda_{\varphi}
\end{array}\right]
$$

calculate them at any time in an extra step via

$$
\begin{aligned}
& \lambda_{n}=\boldsymbol{R}_{d}^{T}\left(\boldsymbol{q}_{n}\right) \cdot \\
& \quad\left[D_{1} L_{d}\left(\boldsymbol{q}_{n}, \boldsymbol{q}_{n+1}\right)+D_{2} L_{d}\left(\boldsymbol{q}_{n-1}, \boldsymbol{q}_{n}\right)+\boldsymbol{f}_{n-1}^{+}+\boldsymbol{f}_{n}^{-}\right]
\end{aligned}
$$

with the $n \times m$ dimensional matrix

$$
\boldsymbol{R}_{d}\left(\boldsymbol{q}_{n}\right)=\left(\boldsymbol{G}_{d}^{A}\left(\boldsymbol{q}_{n}\right)\right)^{T} \cdot\left(\boldsymbol{G}_{d}^{A}\left(\boldsymbol{q}_{n}\right) \cdot\left(\boldsymbol{G}_{d}^{A}\left(\boldsymbol{q}_{n}\right)\right)^{T}\right)^{-1}
$$

see Ref. 10 for further details.

Spherical pair. A spherical joint between two rigid bodies leads to the following $m_{\text {ext }}^{S}=3$ external constraints on the movement of the bodies

$$
\boldsymbol{g}_{\text {ext }}^{S}(\boldsymbol{q})=\varphi^{2}-\varphi^{1}+\rho^{2}-\rho^{1}=0
$$

The related constraint force $\boldsymbol{F}_{\varphi}$ that prevents the bodies from relative displacement is visualised in the free body diagram on the left hand side of Figure 4. For further details on the formulation of the spherical joint connection see for example Refs. 10 and 12 .

With the Jacobian $\boldsymbol{G}_{\text {ext }}^{S}(\boldsymbol{q}) \in \mathbb{R}^{3 \times 24}$ of the external constraints and $\lambda_{\text {ext }}^{S}=\lambda_{\varphi} \in \mathbb{R}^{3}$ representing the

Herein, $\hat{\boldsymbol{a}}$ denotes a skew-symmetric $3 \times 3$ matrix with the corresponding axial vector $a \in \mathbb{R}^{3}$. Considering for example the first body, it is evident that a force $\boldsymbol{h}_{\varphi}^{1}=-\lambda_{\varphi}$ acting in the centre of mass of the body and an additional torque $\boldsymbol{h}_{\theta}^{1}=-\boldsymbol{\rho}^{1} \times \boldsymbol{\lambda}_{\varphi}$ can be equivalently represented by one force $-\lambda_{\varphi}$, whose point of application is given by $\boldsymbol{\rho}^{1}$ in the respective body frame. Together with the illustration of the free body diagram on the left hand side of Figure 4, it is obvious that the constraint force $\boldsymbol{F}_{\varphi}$ acting in the joint and preventing the bodies from relative translational displacement is given by

$$
\boldsymbol{F}_{\varphi}=\lambda_{\varphi}
$$

Cardan pair. Two rigid bodies connected by a cardan joint have two relative rotational degrees of freedom, see right hand part of Figure 4, which leads to a number of $m_{\text {ext }}^{C}=4$ external constraints. Let the two axes of rotation of a cardan joint be

$$
\begin{aligned}
\boldsymbol{n}_{1}\left(\boldsymbol{q}^{1}\right) & =\sum_{i=1}^{3} n_{1 i} \boldsymbol{d}_{i}^{1} \in \mathbb{R}^{3} \text { and } \boldsymbol{n}_{2}\left(\boldsymbol{q}^{2}\right) \\
& =\sum_{i=1}^{3} n_{2 i} \boldsymbol{d}_{i}^{2} \in \mathbb{R}^{3} \text { with } \boldsymbol{n}_{1}\left(\boldsymbol{q}^{1}\right)^{T} \cdot \boldsymbol{n}_{2}\left(\boldsymbol{q}^{2}\right)=0
\end{aligned}
$$

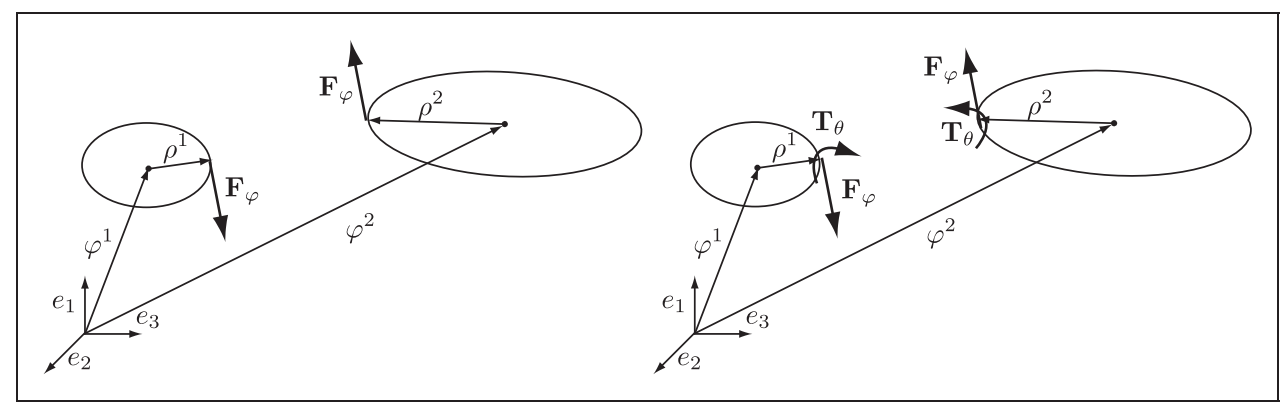

Figure 4. Free body diagram to visualise the constraint forces in a spherical joint (left) and the constraint forces and torques in a cardan joint (right). 
Then, constraining the rotation about the third axis means that these two rotation axes have to stay in their initial orientation to each other

$$
\boldsymbol{g}_{\text {ext }}^{C}(\boldsymbol{q})=\left[\begin{array}{c}
\varphi^{2}-\boldsymbol{\varphi}^{1}+\boldsymbol{\rho}^{2}-\boldsymbol{\rho}^{1} \\
\boldsymbol{n}_{1}\left(\boldsymbol{q}^{1}\right)^{T} \cdot \boldsymbol{n}_{2}\left(\boldsymbol{q}^{2}\right)
\end{array}\right]
$$

With $\boldsymbol{I}_{12}=\boldsymbol{I}-\boldsymbol{n}_{1} \otimes \boldsymbol{n}_{1}-\boldsymbol{n}_{2} \otimes \boldsymbol{n}_{2} \in \mathbb{R}^{3 \times 3}$, where $\otimes$ denotes the dyadic product, the external part of the nullspace matrix reads

$$
\boldsymbol{P}_{e x t}^{2, C}(\boldsymbol{q})=\left[\begin{array}{cccc}
\boldsymbol{I}_{3 \times 3} & \boldsymbol{\rho}^{2}-\boldsymbol{\rho}^{1} \cdot \boldsymbol{I}_{12} & \boldsymbol{\rho}^{2} \times \boldsymbol{n}_{1} & \boldsymbol{\rho}^{2} \times \boldsymbol{n}_{2} \\
\boldsymbol{0} & \boldsymbol{I}_{12} & \boldsymbol{n}_{1} & \boldsymbol{n}_{2}
\end{array}\right]
$$

If the cardan pair is actuated by torques $\tau^{C} \in \mathbb{R}^{2}$, then the actuation forces on each body are computed (similar to equation (42) in Ref. 10) with

$$
\begin{aligned}
& \boldsymbol{C}^{1, C}(\boldsymbol{q})=\left[\begin{array}{cc}
\boldsymbol{0}_{3 \times 1} & \boldsymbol{0}_{3 \times 1} \\
-\boldsymbol{n}_{1} & -\boldsymbol{n}_{2}
\end{array}\right] \text { and } \\
& \boldsymbol{C}^{2, C}(\boldsymbol{q})=\left[\begin{array}{cc}
\boldsymbol{0}_{3 \times 1} & \boldsymbol{0}_{3 \times 1} \\
\boldsymbol{n}_{1} & \boldsymbol{n}_{2}
\end{array}\right]
\end{aligned}
$$

The Lagrange multipliers $\lambda_{e x t}^{C} \in \mathbb{R}^{4}$ consist of $\lambda_{\varphi} \in \mathbb{R}^{3}$ related to the constraints on relative translation of the bodies and $\lambda_{\theta} \in \mathbb{R}$ related to the constrained rotation about the axis perpendicular to $\boldsymbol{n}_{1}$ and $\boldsymbol{n}_{2}$. Consequently, using the Jacobian $\boldsymbol{G}_{\text {ext }}^{C}(\boldsymbol{q}) \in \mathbb{R}^{4 \times 24}$ of the constraints in equation (18), the constraint forces and torques on the centre of mass of the bodies read

$$
\begin{aligned}
{\left[\begin{array}{c}
\boldsymbol{h}_{\varphi}^{1} \\
\boldsymbol{h}_{\theta}^{1} \\
\boldsymbol{h}_{\varphi}^{2} \\
\boldsymbol{h}_{\theta}^{2}
\end{array}\right]=} & \boldsymbol{P}_{\text {int }}^{T} \cdot\left(\boldsymbol{G}_{\text {ext }}^{C}\right)^{T} \cdot \lambda_{\text {ext }}^{C} \\
= & {\left[\begin{array}{c}
-\lambda_{\varphi} \\
-\boldsymbol{\rho}^{1} \times \lambda_{\varphi}+\lambda_{\theta}\left(\boldsymbol{n}_{1} \times \boldsymbol{n}_{2}\right) \\
\lambda_{\varphi} \\
\boldsymbol{\rho}^{1} \times \lambda_{\varphi}-\lambda_{\theta}\left(\boldsymbol{n}_{1} \times \boldsymbol{n}_{2}\right)
\end{array}\right] }
\end{aligned}
$$

Taking into account the free body diagram of a cardan joint (see right hand part of Figure 4), we obtain the constraint force in a cardan joint $\boldsymbol{F}_{\varphi}$ that prevents the bodies from relative translations and the resulting constraint torque $\boldsymbol{T}_{\theta}$ preventing relative rotation about the axis $\boldsymbol{n}_{1} \times \boldsymbol{n}_{2}$ via

$$
\boldsymbol{F}_{\varphi}=\lambda_{\varphi} \text { and } \boldsymbol{T}_{\theta}=\lambda_{\theta}\left(\boldsymbol{n}_{1} \times \boldsymbol{n}_{2}\right)
$$

Kinematic chain representing a human arm. For the simulation of typical human arm movements, we represent the arm as a kinematic chain, see left hand part of Figure 5. We investigate a multibody system consisting of three bodies connected via three joints as depicted in the right part of Figure 5. The shoulder is modelled as a spherical joint and is fixed in space in a point $\boldsymbol{X}_{G} \in \mathbb{R}^{3}$. The joints representing elbow and wrist are modelled as cardan joints. Combining the constraints of spherical and cardan joint connections (equations (16) and (18)), this results in $m_{e x t}^{A}=m_{e x t}^{S}+2 m_{e x t}^{C}=11$ external constraints on the system. The axes of rotation are denoted as $\boldsymbol{n}_{1}^{1}\left(\boldsymbol{q}^{1}\right), \boldsymbol{n}_{2}^{1}\left(\boldsymbol{q}^{2}\right)$ and $\boldsymbol{n}_{1}^{2}\left(\boldsymbol{q}^{2}\right), \boldsymbol{n}_{2}^{2}\left(\boldsymbol{q}^{3}\right)$ in the first and second cardan joint, respectively

$$
\boldsymbol{g}_{\text {ext }}^{A}(\boldsymbol{q})=\left[\begin{array}{c}
\boldsymbol{\varphi}^{1}+\boldsymbol{\rho}^{11}-X_{G} \\
\varphi^{2}+\boldsymbol{\rho}^{21}-\boldsymbol{\varphi}^{1}-\boldsymbol{\rho}^{12} \\
\boldsymbol{n}_{1}^{1}\left(\boldsymbol{q}^{1}\right)^{T} \cdot \boldsymbol{n}_{2}^{1}\left(\boldsymbol{q}^{2}\right) \\
\varphi^{3}+\boldsymbol{\rho}^{31}-\boldsymbol{\varphi}^{2}-\boldsymbol{\rho}^{22} \\
\boldsymbol{n}_{1}^{2}\left(\boldsymbol{q}^{2}\right)^{T} \cdot \boldsymbol{n}_{2}^{2}\left(\boldsymbol{q}^{3}\right)
\end{array}\right] \in \mathbb{R}^{11}
$$

Corresponding to the number of constraints, $m_{e x t}^{A}=11$ Lagrange multipliers are present that are arranged as follows

$$
\lambda_{e x t}^{A}=\left[\begin{array}{lllll}
\lambda_{\varphi}^{1} & \lambda_{\varphi}^{2} & \lambda_{\theta}^{2} & \lambda_{\varphi}^{3} & \lambda_{\theta}^{3}
\end{array}\right]^{T} \in \mathbb{R}^{11}
$$

With the Jacobian of the constraints denoted by $\boldsymbol{G}_{\text {ext }}^{A}(\boldsymbol{q}) \in \mathbb{R}^{11 \times 36}$, we calculate a set of constraint

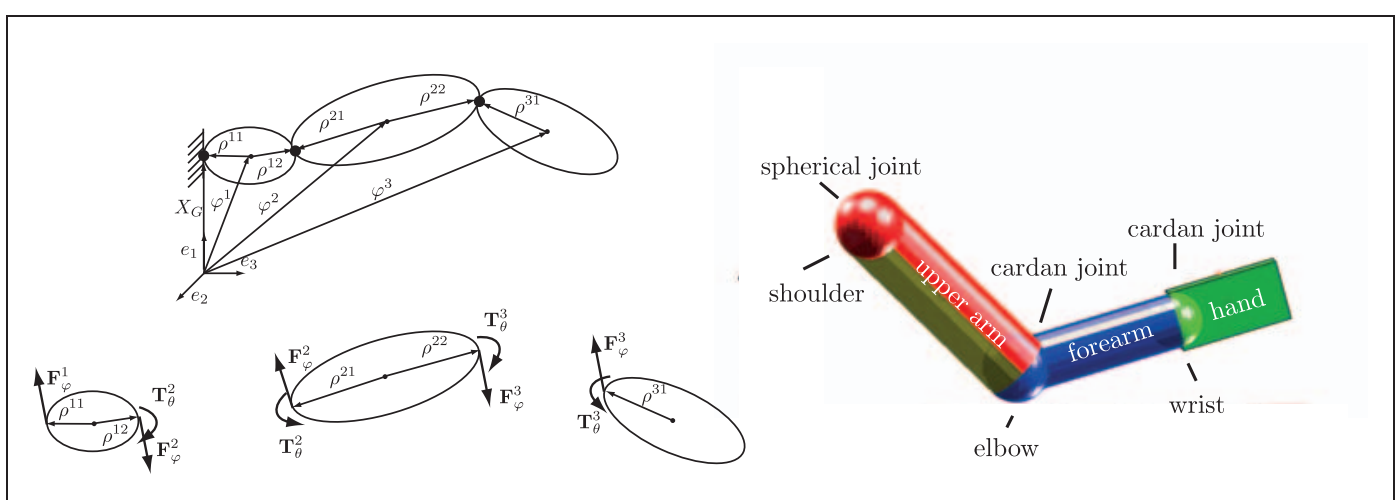

Figure 5. Kinematic chain representing an arm with free body diagram (left) and rigid body model of a human arm (right). 
forces, which can be transformed, as illustrated above, to a set of constraint forces and torques in the centre of mass of each body

$$
\left[\begin{array}{c}
\boldsymbol{h}_{\varphi}^{1} \\
\boldsymbol{h}_{\theta}^{1} \\
\boldsymbol{h}_{\varphi}^{2} \\
\boldsymbol{h}_{\theta}^{2} \\
\boldsymbol{h}_{\varphi}^{3} \\
\boldsymbol{h}_{\theta}^{3}
\end{array}\right]=\left[\begin{array}{c}
\lambda_{\varphi}^{1}-\lambda_{\varphi}^{2} \\
\widehat{\boldsymbol{\rho}^{11}} \cdot \lambda_{\varphi}^{1}-\widehat{\boldsymbol{\rho}^{12}} \cdot \boldsymbol{\lambda}_{\varphi}^{2}+\lambda_{\theta}^{2}\left(\boldsymbol{n}_{1}^{1} \times \boldsymbol{n}_{2}^{1}\right) \\
\lambda_{\varphi}^{2}-\lambda_{\varphi}^{3} \\
\widehat{\boldsymbol{\rho}^{21}} \cdot \lambda_{\varphi}^{2}-\widehat{\boldsymbol{\rho}^{22}} \cdot \lambda_{\varphi}^{3}-\lambda_{\theta}^{2}\left(\boldsymbol{n}_{1}^{1} \times \boldsymbol{n}_{2}^{1}\right)+\lambda_{\theta}^{3}\left(\boldsymbol{n}_{1}^{2} \times \boldsymbol{n}_{2}^{2}\right) \\
\lambda_{\varphi}^{3} \\
\widehat{\boldsymbol{\rho}^{31}} \cdot \lambda_{\varphi}^{3}-\lambda_{\theta}^{3}\left(\boldsymbol{n}_{1}^{2} \times \boldsymbol{n}_{2}^{2}\right)
\end{array}\right]
$$

A similar consideration as for the spherical and cardan pair yields the constraint forces and torques acting in the single joints, as illustrated in the free body diagram in Figure 5. The constraint force $\boldsymbol{F}_{\varphi}^{\mathbf{1}}$ prevents the first body (upper arm) from translation relative to the point $\boldsymbol{X}_{G}$, where it is fixed in space, $\boldsymbol{F}_{\varphi}^{2}$ prevents the forearm from translational movements relative to the upper arm and the hand is prevented from relative translation with respect to the forearm via $\boldsymbol{F}_{\varphi}^{3}$

$$
\boldsymbol{F}_{\varphi}^{1}=\lambda_{\varphi}^{1}, \quad \boldsymbol{F}_{\varphi}^{2}=\lambda_{\varphi}^{2}, \quad \boldsymbol{F}_{\varphi}^{3}=\lambda_{\varphi}^{3}
$$

The constraint torques $\boldsymbol{T}_{\theta}^{2}$ and $\boldsymbol{T}_{\theta}^{3}$ that constrain the rotation about one axis in the elbow and the wrist, can be calculated via

$$
\boldsymbol{T}_{\theta}^{2}=\lambda_{\theta}^{2}\left(\boldsymbol{n}_{1}^{1} \times \boldsymbol{n}_{2}^{1}\right) \quad \boldsymbol{T}_{\theta}^{3}=\lambda_{\theta}^{3}\left(\boldsymbol{n}_{1}^{2} \times \boldsymbol{n}_{2}^{2}\right)
$$

\section{Muscles around the human elbow}

To make the simulations more realistic, the actuation of the elbow is replaced by Hill-type muscle actuation. We therefore implement the seven most important muscles around the elbow with muscle data according to Refs. 15 and 16, which has to be changed slightly to ensure that the $f_{l}$ and $f_{v}$ values stay in a reasonable range. In Figure 6, the paths of these muscles around the elbow are shown. Herein, the black nodes are

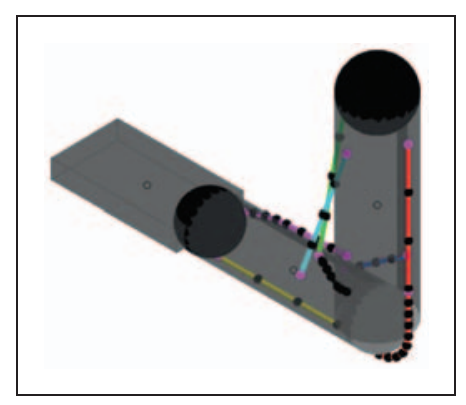

Figure 6. Muscle paths around the elbow for the seven most important arm muscles. check points to ensure that the calculated path does not intersect the rigid bodies. Triceps and Biceps are the most commonly known muscles, which are depicted in red and green in the example. They contribute to flexion and extension of the elbow, together with the brachialis muscle (depicted in cyan). Musculus suplinator (blue) and pronator (black) are mostly responsible for suplination (palm up) and pronation (palm down) movements. In addition, the musculus extensor carpi radialis longus (yellow) and the musculus brachioradialis (magenta) contribute to elbow motion.

\section{Numerical examples}

\section{Optimal control of steering motion with joint actuation}

For the example of an arm performing a steering motion, the optimal control problem can be formulated according to equation (7). Additional nonlinear equality constraints for this example result from prescribing a rest-to-rest manoeuvre. Moreover, the initial configuration is predefined $\left(\boldsymbol{u}\left(t_{0}\right)=\boldsymbol{u}_{0}\right)$ as well as the final location of the hand via $\boldsymbol{x}_{h}\left(t_{N}\right)=\boldsymbol{x}_{h N}$. During the motion, the hand has to follow a circular path representing the steering wheel. This is implemented via constraining the location of the hand $\boldsymbol{h}_{d}\left(\boldsymbol{u}_{n}, \boldsymbol{u}_{n+1}, \tau_{n}\right)=\boldsymbol{0}$ in every time step. The total motion of the hand describes a quadrant on the wheel during $t_{N}=1.04 \mathrm{~s}$ simulation time with a step size of $\Delta t=0.04 \mathrm{~s}$, while gravity is present. Further on, inequality constraints are present that impose limits on the possible joint angles according to the anatomical restrictions in the shoulder, the elbow and the wrist. Beyond that, additional inequality constraints are formulated to keep the palm oriented inwards. Generally, no bounds are set to configuration and torque values, except for the simulation using the constraint force criterion, here the joint torques are limited to stay between $-20 \mathrm{Nm}$ and $+20 \mathrm{Nm}$.

The upper left diagram in Figure 7 shows the resulting optimal torques in one direction of the shoulder joint. This direction is exemplarily shown for all torques, as the largest amount of torque occurs here. One can see in this diagram that the control effort, which is represented by the area under these curves, is minimal for the trajectory created with the minimal control effort criterion and the difference to the result of the minimum torque change criterion is only slight. Likewise, it is obvious that the minimum torque change criterion leads to the smoothest evolution of torques. From the evolution of kinetic energy over time (Figure 7, top right), it becomes clear that the kinetic energy of the motion can be evidently reduced using a minimum kinetic energy criterion. In the lower left diagram in Figure 7 , the norm of the constraint force in the 


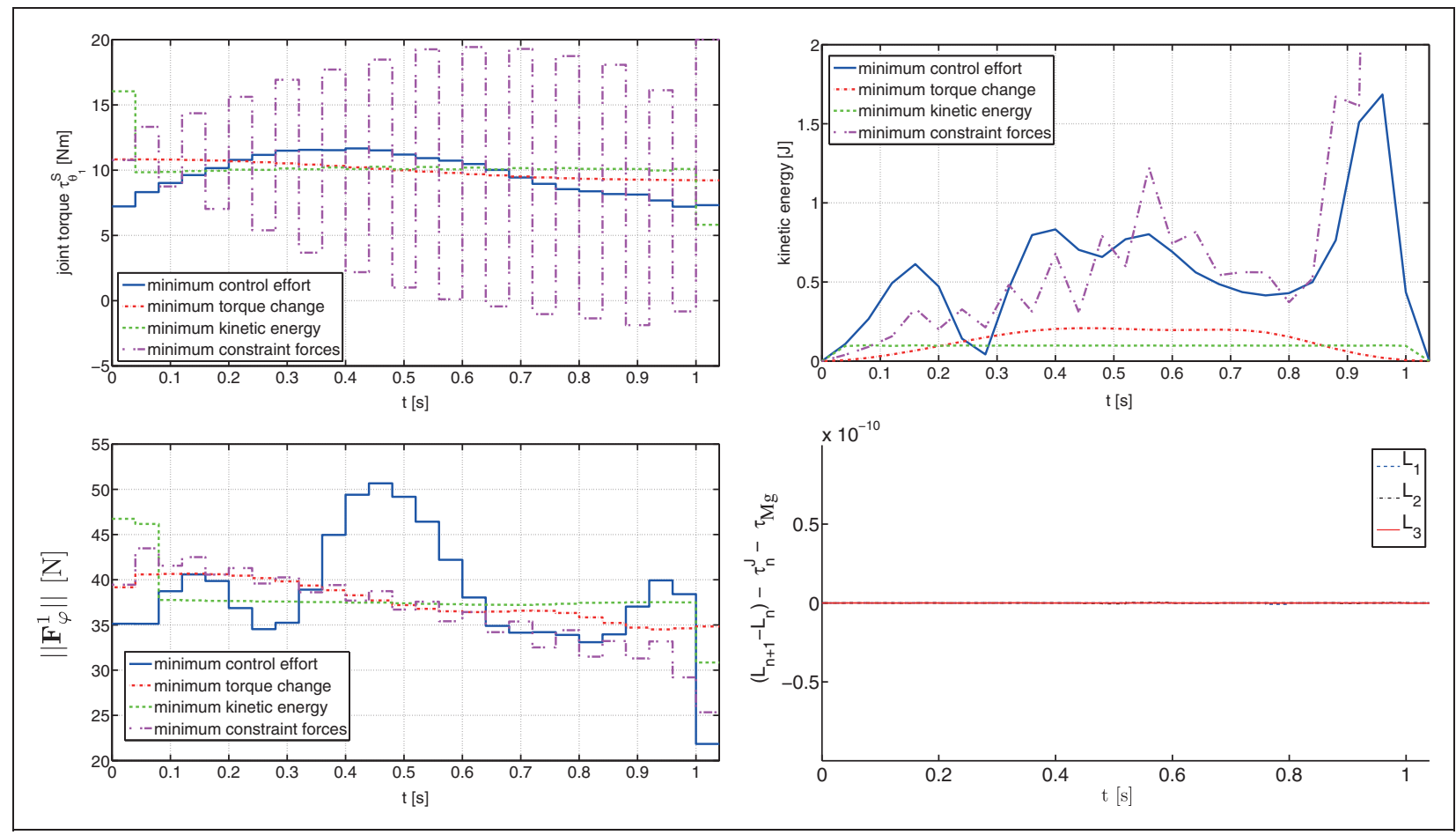

Figure 7. Results of optimal control simulations with different cost functions. Evolution of the first component of the torques in the shoulder $\tau_{\theta_{1}}^{l}$ over time (top left), evolution of the kinetic energy over time (top right), evolution of the norm of the constraint force $\boldsymbol{F}_{\varphi}^{\prime}$ in the shoulder over time (bottom left) and consistency of angular momentum (bottom right).

shoulder is exemplarily depicted. Here, the cost function minimising the constraint forces naturally leads to the curve with the lowest area below. However, this criterion leads to a relatively large control effort with alternating torque evolutions over time, see upper left diagram in Figure 7. A numerical verification of angular momentum consistency (see Remark in section 'Structure preserving discrete equations of motion') is shown in Figure 7 bottom right. The angular momentum changes exactly according to the actuating torques and those resulting from gravity.

When observing the motion itself, which is illustrated in Figure 8 for four distinct time nodes, it is obvious that the criterion for minimum control effort does not lead to a realistic steering motion, as it seems that there is a tendency of gaining momentum during the first time steps, see Figure 8, left column. Something similar shows up for the minimum constraint forces criterion, which leads to unrealistic relative rotation between upper arm and forearm in the end of the motion (see Figure 8 at the bottom right). The criteria of minimum torque change and minimum kinetic energy both lead to realistic motion and reasonable evolutions of torques. Movies of the motions are available at http://www.ltd.tf.uni-erlangen.de/ Research/Research.htm.

Remark. As DMOCC uses in this example a solver based on 'sqp' (SNOPT7 for Matlab), which provides only local solutions that strongly depend on the initial guess, see Ref. 10, all simulations are started with the same initial guess created by a forward dynamics simulation with initial velocities, to make the results comparable. The problem of finding global solutions is a future challenge. Further on, the focus of this example is not on minimal CPU time, as no analytical gradients are provided. On average the CPU time lies between $2 \mathrm{~h}$ and one day. The solution for the minimum constraint forces criterion is performed with a feasibility and optimality tolerance of $10^{-6}$, all other calculations are performed with a feasibility and optimality tolerance of $10^{-8}$.

\section{Optimal control of lifting the arm with muscle actuation}

In a second example, the arm is supposed to lift from an initial vertical position to a flexed position. Again, the optimal control problem can be formulated according to equation (7). As in the first example, a rest-to-rest manoeuvre is simulated, which leads to constraints on the initial and final momentum being zero. Further on, the initial configuration is predefined $\left(\boldsymbol{u}\left(t_{0}\right)=\boldsymbol{u}_{0}\right)$ as well as the final location of the hand's centre of mass via $\boldsymbol{x}_{h}\left(t_{N}\right)=\boldsymbol{x}_{h N}$. The activities of the muscles are restricted to $\boldsymbol{A}_{n} \in[0,1]$ for $n=1, \ldots, N-1$, and bounds on the joint torques are set at $\pm 5 \mathrm{~N}$. By further bounds on the configuration variables, unrealistic joint angles are avoided. The simulation time is $t_{N}=0.52 \mathrm{~s}$ with a step size of $\Delta t=0.02 \mathrm{~s}$, while gravity is present. In contrast to the first example, the joint torques in the elbow are replaced by seven Hill-type muscle models representing the actuation of the most important 


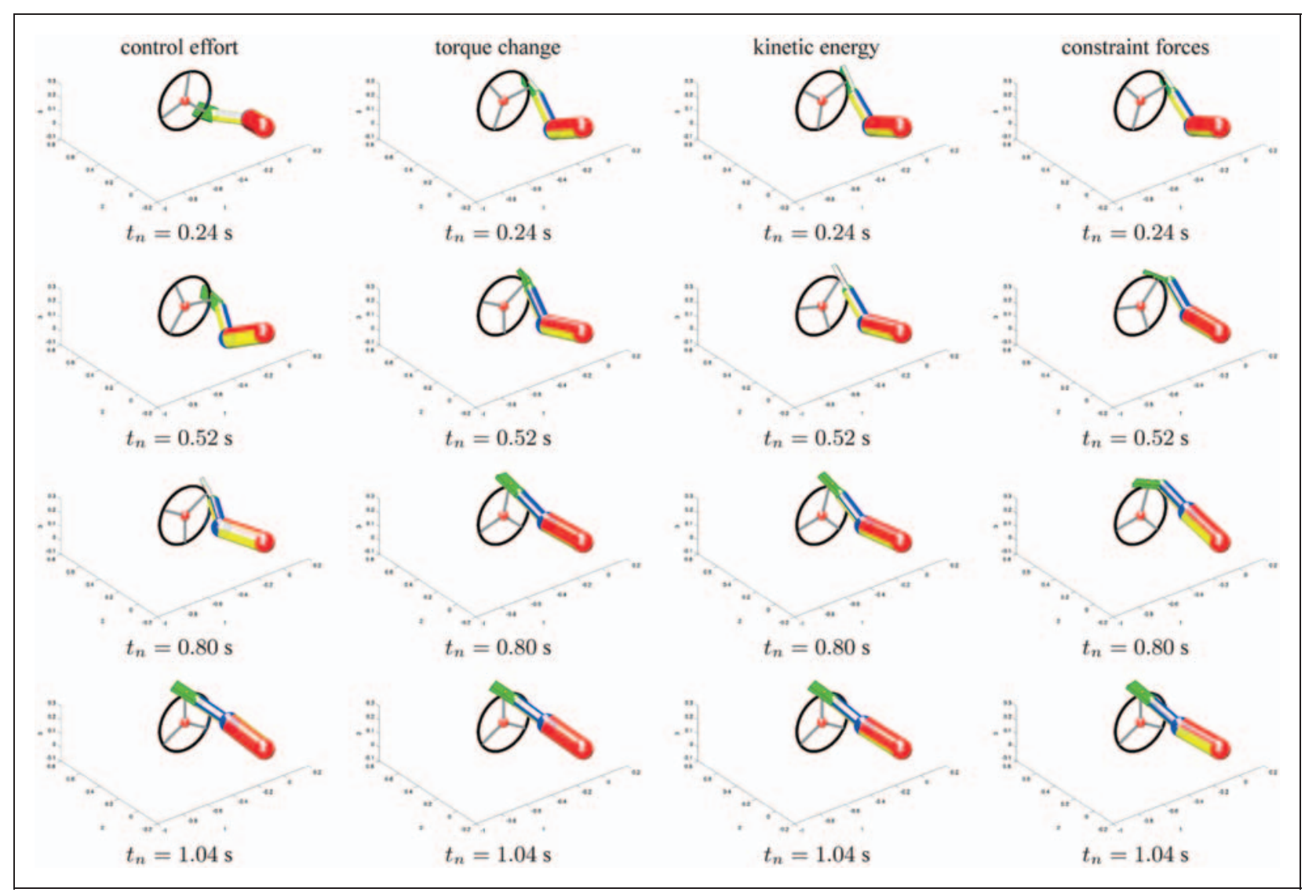

Figure 8. Resulting motion of optimal control simulations at four distinct time nodes for minimum control effort (left column), minimum torque change (second column), minimum kinetic energy criterion (third column) and minimum constraint forces criterion (right column).

elbow muscles. Hence, the optimisation variables contain additionally the activities of the seven muscles in each time step, replacing the torques in the elbow. This leads to the typical redundancy of biomechanical problems, where usually more muscles are involved than degrees of freedom are present. Furthermore, analytical gradients of the cost function and the constraints are implemented, which naturally increases performance and accuracy. We compare several cost functions that minimise certain aspects of the motion and choose Matlab's fmincon solver with algorithm option 'interior-point'. Especially for cost functions that are highly nonlinear in the optimisation variables, it seems that the interior-point algorithm shows a better performance than for cost functions that are linear or quadratic in the optimisation variables. In particular, if the solution lies close to the boundary of the admissible set, the 'sqp' algorithm finds a similar local minimum. This effect has to be investigated in future work to decide which algorithm type is appropriate for simulations with muscles. Several initial guesses are used for this example and for comparability, only the best solutions, achieved with the interior-point algorithm, are reported. We requested a feasibility and optimality tolerance of $10^{-8}$ and all simulations finished with exitflag 2, meaning a local minimum is possible, even if the first-order optimality is not smaller that the optimality tolerance. In particular, for the constraint force criterion, this is an issue. The CPU times for these simulations lie between 30 and $120 \mathrm{~min}$.

Due to the implementation of muscle models, we can directly involve muscle-related quantities like activities or the resulting muscle forces into the cost function. One can for example ask for a motion with minimal muscle activities (together with minimal torques in the other joints), see equation (12), or for a motion with minimal changes in the muscle activities and joint torques, see equation (13), similar to the criterion of minimal torque change in the first example without muscles. Even the muscle forces can be involved and minimising the muscle force effort (again together with minimal torques in the other joints) can be the goal, see equation (14). Further on, weighting the muscle forces by the cross-sectional area of the muscles to calculate a stress in the muscle is possible. Minimising this muscle stress criterion, see equation (15), probably leads to a motion where the muscles with larger cross-sectional area work more than thinner muscles. The values of the cross-sectional areas are taken from Ref. 16. According to Ref. 26, this criterion leads to a motion that can be performed with maximal endurance. A key challenge for all cost functions is to find appropriate weighting factors 


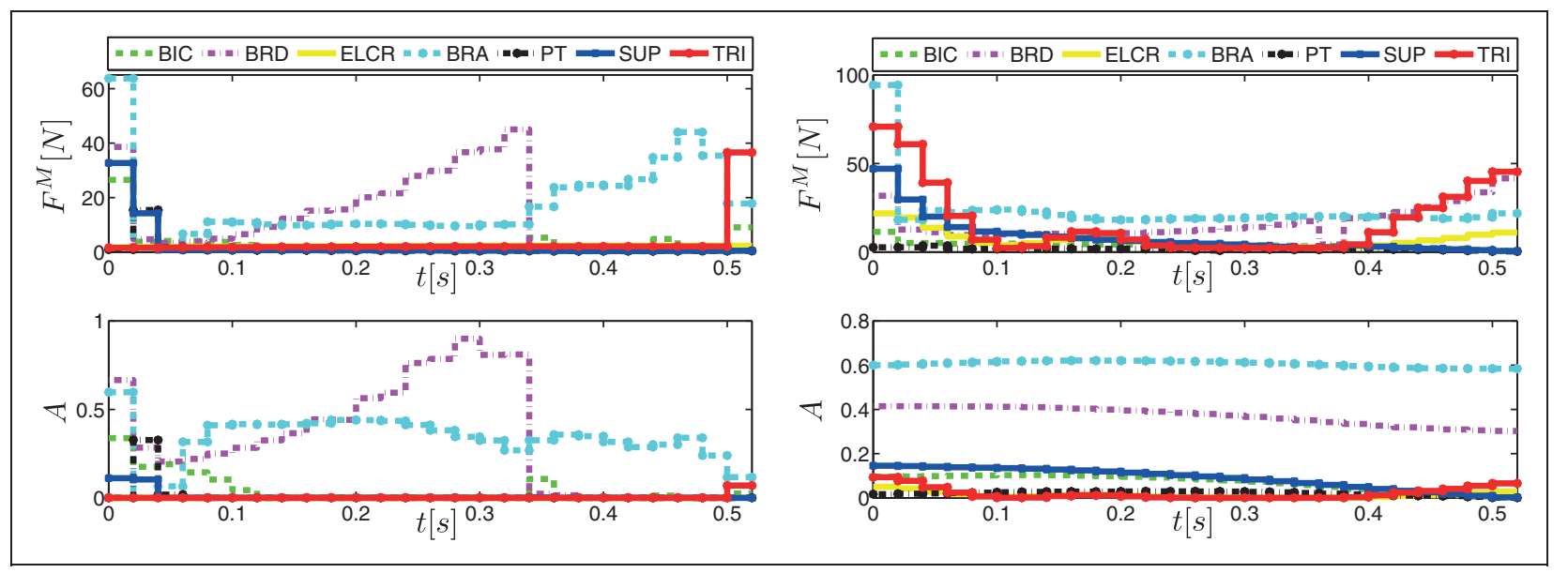

Figure 9. Activities and muscle forces of the elbow muscles while lifting the arm for a minimum activities and joint torques criterion (I2) (left) and for a criterion on minimum change in activities and joint torques (I3) (right).

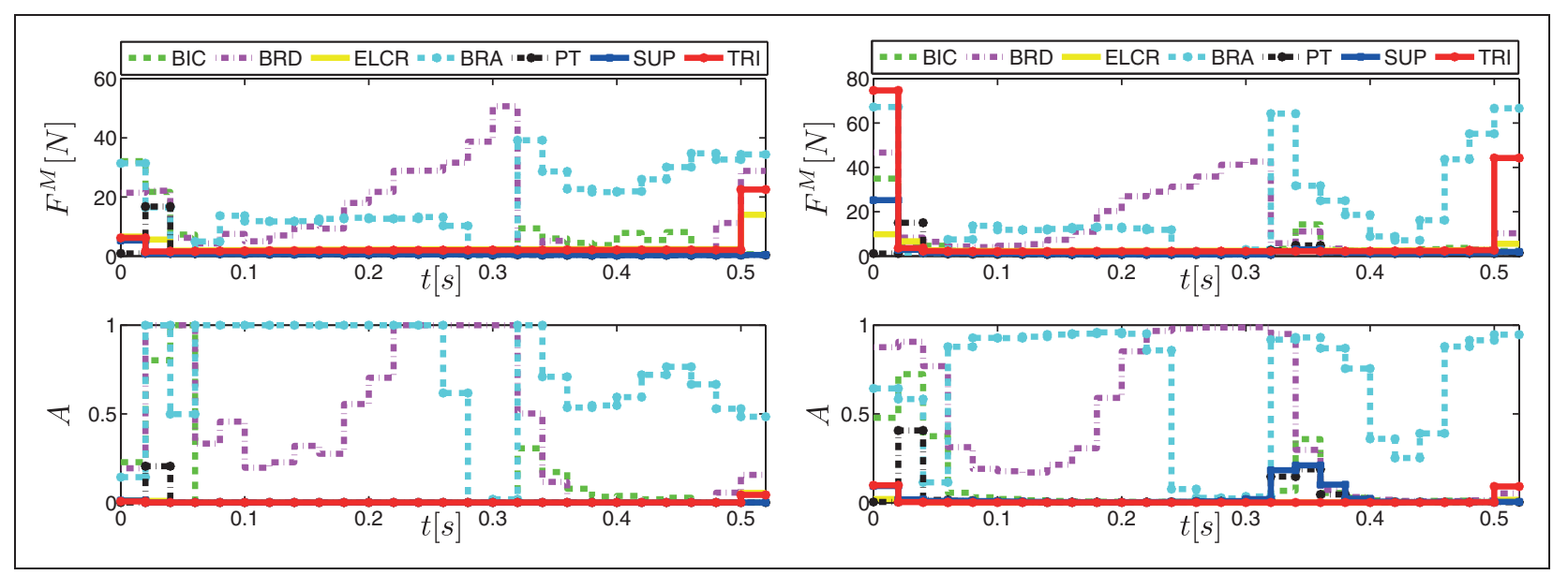

Figure 10. Activities and muscle forces of the elbow muscles while lifting the arm for a minimum muscle forces and joint torques criterion (14) (left) and for a criterion on minimum muscle stress and joint torques (I5) (right).

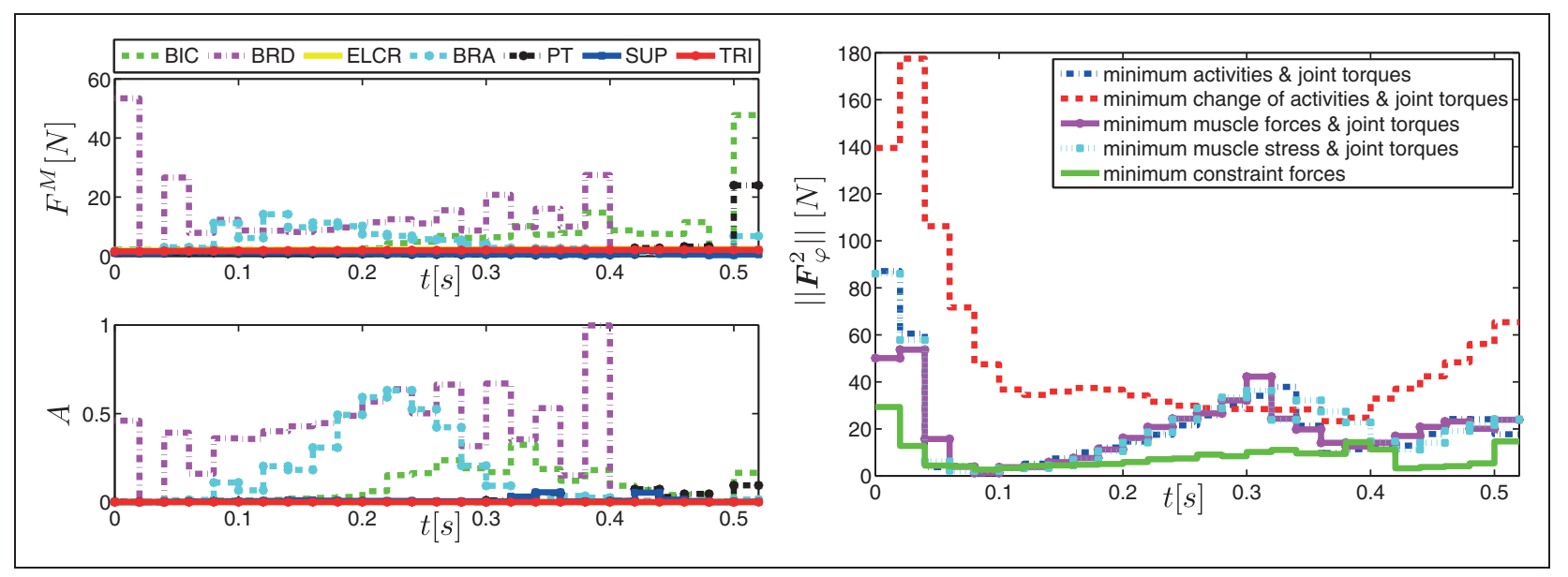

Figure II. Activities and muscle forces of the elbow muscles while lifting the arm for a minimum constraint forces criterion (II) (left) and the norm of the constraint force in the elbow $\left(F_{\varphi}^{2}\right)$ for all criteria (right). 
to get reasonable results, where both the muscle forces or activities and the joint torques in shoulder and wrist are optimised.

For the criterion of minimal activities and torques (see equation (12)), the resulting muscle activities and forces are depicted in the left hand part of Figure 9. Obviously, the activities of the muscles are reduced, compared to the resulting activities when using other criteria, see right hand part of Figure 9 and both plots in Figure 10. Like for all other criteria, the main part of muscle force is coming from musculus brachialis and musculus brachidorialis, which is also reported in anatomy books like Ref. 27. The musculus biceps is also involved during the first part of the motion but it produces only a small amount of force. This may have a reason in the chosen insertion points, which cannot be absolutely realistic because of the simplified elbow mechanics in our model. However, it is frequently reported ${ }^{27-29}$ that the musculus brachialis is the strongest muscle in elbow flexion and the

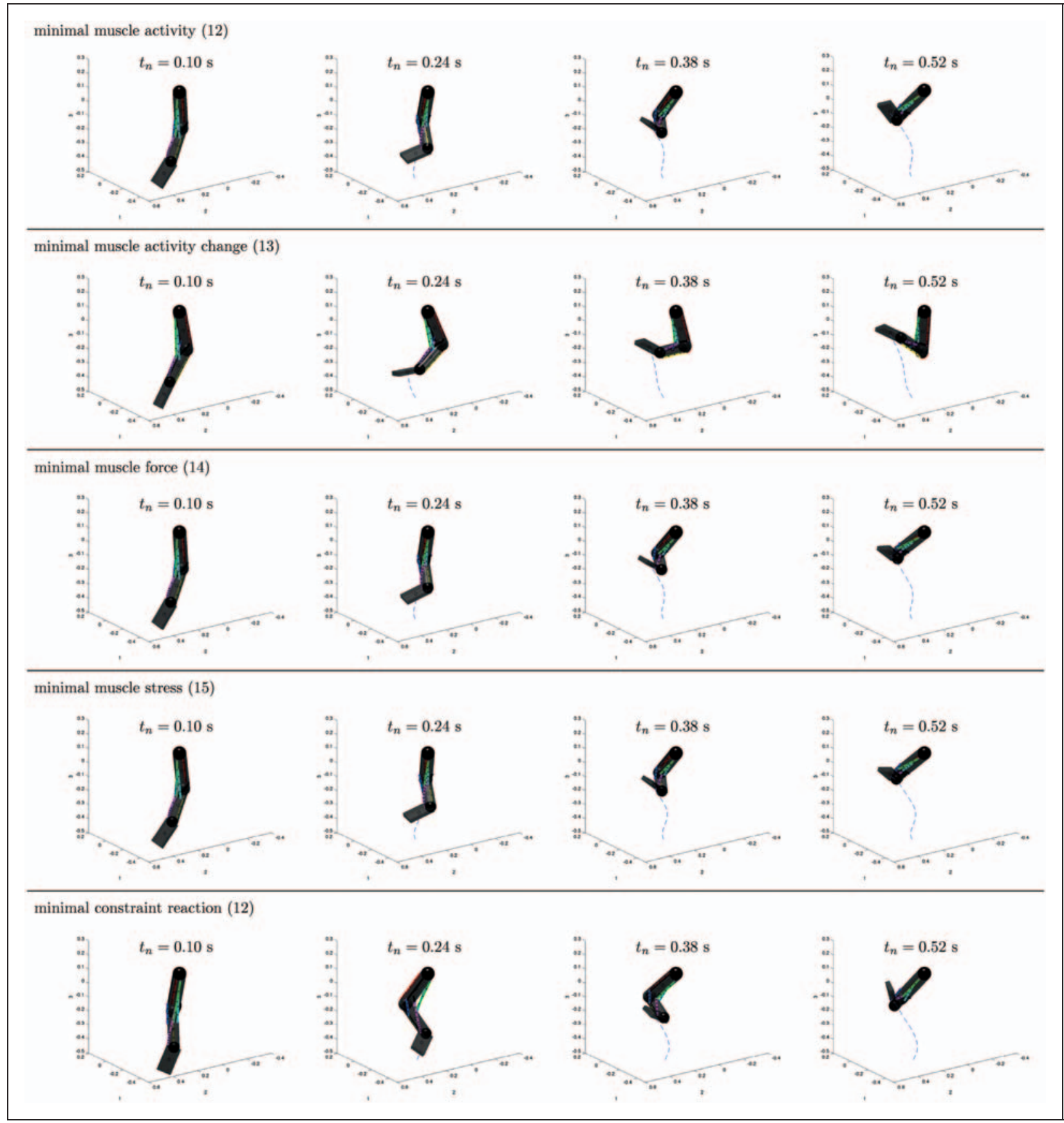

Figure 12. Resulting arm motions from the optimal control simulations: minimal activities and joint torques (equation (I2)), minimal change in activities and joint torques (equation (13)), minimal muscle forces and joint torques (equation (14)), minimal muscle stresses and joint torques (equation (I5)) and minimal constraint reactions (equation (II)) (from top to bottom). Movies of the motions are available at http://www.Itd.tf.uni-erlangen.de/Research/Research.htm. 
musculus biceps is more responsible for pronation and suplination movements, which will be investigated in future work.

In the right hand part of Figure 9, the resulting activities and muscle forces are illustrated for the criterion of minimal changes in activities and joint torques (see equation (13)). The evolution of activities is nearly constant, which apparently yields a minimal change. This criterion leads to higher muscle forces in contrast to the other results and especially musculus triceps is involved at the beginning and end of the motion.

When minimising the muscle forces and the joint torques (equation (14)), see left hand part of Figure 10 , muscle forces are reduced, yet they are comparable to the result of minimal activities. However, for this criterion the muscle activities are relatively high at time steps, where force-length $\left(f_{l}\right)$ or force-velocity $\left(f_{v}\right)$ factors are rather low. There, a high activity has nearly no influence on the resulting muscle force. In this case, there is no need for the solver to reduce an activity, which leads to mostly random activities if $f_{l}$ or $f_{v}$ are close to zero.

If the muscle forces are weighted by their crosssectional area and this resulting muscle stress is minimised together with the joint torques (see equation (15)), this yields muscle activities as illustrated in the right hand part of Figure 10. The musculus triceps with the largest cross-sectional area is involved more, in particular at the beginning and end of the motion and the musculus brachialis undertakes some part of the force for the musculus brachioradialis, which has a smaller cross-sectional area.

The right hand part of Figure 11 shows the evolution of the norm of the constraint force in the elbow. The amount of constraint force is obviously reduced when using the minimum constraint force criterion. In particular, at the beginning and end of the motion larger muscle forces arise, while during the mid time of the motion, only small muscle forces are necessary, as can be seen in the left hand part of Figure 11. However, the high torque effort of the motion (alternating as in the first example without muscles) increases the total effort of the motion to a value higher than that of the minimised muscle force and joint torques criterion.

The snapshots of the motions obtained from the optimal control simulations with the different criteria (Figure 12) show comparable final configurations for the criteria of minimal activities and joint torques, minimal muscle forces and joint torques as well as for minimal muscle stresses and joint torques. Note that for all simulations only the final position of the hand's centre of mass is prescribed. However, the whole motions slightly differ, which can also be examined from movies of the motions available at http://www.ltd.tf.uni-erlangen.de/Research/Research. $\mathrm{htm}$. The motion and end configuration for the minimal change in activities and joint torques criterion and the minimal constraint forces criterion differ substantially from the others. Both trajectories of the hand seem rather smooth, while minimising the constraint forces yields a considerable lateral motion of the elbow and the minimal change in activities and joint torques yields the motion which is subjectively looking most natural.

\section{Conclusions}

The focus of this study is on the optimal control of biomechanical motion with appropriate optimal control criteria and cost functions. The optimal control problems are solved using DMOCC, yielding the benefit that the resulting optimal discrete trajectories exactly represent certain characteristics of the real system like for example consistency of momentum maps and symplecticity. In detail, this means that all applied forces and joint torques are exactly translated into motion of the system and every change in angular momentum results only and exactly from the applied forces and torques. This is what clearly distinguishes our work from other investigations that use typical commercial available integrators suffering from numerical dissipation or gain in energy or angular momentum.

Several well-known optimal control criteria are investigated within the used rigid body formulation. A further idea to formulate a criterion taking into account the impact on the joints during the motion is introduced. Herein, the constraint forces that occur in the joints are minimised. Due to the formulation of an index 3 system, the constraint forces acting in the joints can be directly accessed. The computation of the constraint forces is illustrated for a spherical and a cardan pair, as well as for a kinematic chain consisting of three bodies and joints. Further on, this kinematic chain is used to represent a human arm. Finally, the optimal control problem is exemplarily solved for the arm model performing a steering motion. Using all introduced cost functions, the resulting trajectories and motions are compared with particular focus on the results of the criterion that minimises the impact on the joints. In this first attempt, the minimum constraint forces criterion reduces them substantially compared to the other cost functions, but it also leads to a large control effort with alternating torque evolutions. The minimum control effort criterion yields minimal torques but an unrealistic steering motion, whereas both the criteria of minimum kinetic energy and minimum torque change seem to perform well.

An optimal control simulation with muscle forces is shown in a second numerical example. Here further criteria are tested, which involve both muscle parameters of the muscles around the elbow and joint torques in shoulder and wrist. Simulations with muscles always imply a redundancy problem, meaning that more muscles than degrees of freedom are present. 
When using an optimal control framework as illustrated in this work, this redundancy problem is solved along the way, yielding different muscle activity behaviour for the different optimal control criteria. Concluding, with a criterion that minimises the change in activities and joint torques, the subjectively most natural looking motion can be achieved, yet with a larger muscle force effort compared to the other criteria. The constraint force criterion results in a more lateral motion of the elbow reducing the impact on the elbow substantially.

Future studies are necessary to evaluate the effect of different optimisation algorithms like 'sqp' or 'barrier' methods. Another challenge is to simulate more complicated motions, where ergonomical aspects play an important role, for example the simulation of assembling processes. Moreover, as shown in Ref. 10 for multibody systems without muscles and in Ref. 30 with one muscle, sports motions like throwing will be investigated with the actuation by seven muscles in the elbow.

\section{Funding}

The support of the German Research Foundation (DFG) is gratefully acknowledged [grant number LE 1841/2-1].

\section{References}

1. Xiang Y, Arora J and Abdel-Malek K. Physics-based modeling and simulation of human walking: a review of optimization-based and other approaches. Struct Multidiscip Optim 2010; 42: 1-23.

2. Ackermann M and Schiehlen W. Dynamic analysis of human gait disorder and metabolical cost estimation. Arch Appl Mech 2006; 75: 569-594.

3. Pandy MG, Zajac FE, Sim E, et al. An optimal control model for maximum-height human jumping. $J$ Biomech 1990; 123: 1185-1198.

4. Stelzer M and von Stryk O. From robots to humans: towards efficient forward dynamics simulation and optimization exploiting structure and sensitivity information. In: Multibody dynamics: ECCOMAS. Dordrecht: Springer, 2005, pp.1-18.

5. Uno Y, Kawato M and Suzuki R. Formation and control of optimal trajectory in human multijoint arm movement. Biol Cybern 1989; 61: 89-101.

6. Flash $\mathrm{T}$ and Hogans N. The coordination of arm movements: an experimentally confirmed mathematical model. J Neurosci 1985; 5: 1688-1703.

7. Friedman $\mathrm{J}$ and Flash T. Trajectory of the index finger during grasping. Exp Brain Res 2009; 196: 497-509.

8. Rosenbaum DA, Loukopoulos LD, Meulenbroek RGJ, et al. Planning reaches by evaluating stored postures. Psychol Rev 1995; 102: 28-67.

9. Rosenbaum DA, Meulenbroek RGJ, Vaughan J, et al. Posture-based motion planning: Applications to grasping. Psychol Rev 2001; 108: 709-734.

10. Leyendecker S, Ober-Blöbaum S, Marsden JE, et al. Discrete mechanics and optimal control for constrained systems. Optim Contr Appl Meth 2010; 31: 505-528.
11. Betsch P and Leyendecker S. The discrete null space method for the energy consistent integration of constrained mechanical systems. Part II: Multibody dynamics. Int J Numer Meth Eng 2006; 67: 499-552.

12. Leyendecker S, Marsden JE and Ortiz M. Variational integrators for constrained dynamical systems. Z Angew Math Mech 2008; 88: 677-708.

13. Maas R, Siebert T and Leyendecker S. On the relevance of structure preservation to simulations of muscle actuated movements. Biomech Model Mech 2012; 11: 543-556.

14. Maas R and Leyendecker S. Optimal control of biomechanical motion using physiologically motivated cost functions. In: The 2nd joint international conference on multibody system dynamics, Stuttgart, Germany, May 29-June 1, 2012.

15. Murray WM, Delp SL and Buchanan TS. Variation of muscle moment arms with elbow and forearm position. J Biomech 1995; 28: 513-525.

16. Murray WM, Buchanan TS and Delp SL. The isometric functional capacity of muscles that cross the elbow. $J$ Biomech 2000; 33: 943-952.

17. Ian Stavness MS and Delp S. A general approach to muscle wrapping over multiple surfaces. In: American society for biomechanics conference, Gainesville, 15-18 August 2012.

18. Winkelmann A, Kirsch J, May CA, et al. Taschenlehrbuch Anatomie. Stuttgart: Thieme, 2010.

19. Geyer H and Herr H. A muscle-reflex model that encodes principles of legged mechanics produces human walking dynamics and muscle activities. IEEE Trans Neural Syst Rehabil Eng 2010; 18: 263-273.

20. Geyer H, Seyfarth A and Blickhan R. Positive force feedback in bouncing gaits. Proc Biol Sci 2003; 270: 2173-2183.

21. Marsden JE and West M. Discrete mechanics and variational integrators. Acta Numer 2001; 10: 357-514.

22. Hill AV. The heat of shortening and the dynamic constants of muscle. Proc R Soc B 1938; 126: 136-195.

23. Siebert T, Rode C, Herzog W, et al. Nonlinearities make a difference: comparison of two common Hill-type models with real muscle. Biol Cybern 2008; 98: 133-143.

24. van Soest AJ and Bobbert MF. The contribution of muscle properties in the control of explosive movements. Biol Cybern 1993; 69: 195-204.

25. Sherman M. How to compute muscle moment arm using generalized coordinates, http://wiki.simtk.org/ opensim/ (2010, accessed 25 April 2013).

26. Crowninshield RD and Brand RA. A physiologically based criterion of muscle force prediction in locomotion. J Biomech 1981; 14: 793-801.

27. Tillmann BN. Atlas der Anatomie des Menschen. Berlin: Springer-Verlag, 2005.

28. Brachialis muscle- wikipedia, http://en.wikipedia.org/ wiki/Brachialis_muscle (2013, accessed 17 April 2013).

29. Musculus brachialis - DocCheck Medical Services, http://flexikon.doccheck.com/de/Musculus_brachialis (2013, accessed 17 April 2013).

30. Ober-Blöbaum S and Timmermann J. Optimal control for a pitcher's motion modeled as constrained mechanical system. In: Proceedings of the 7th international conference on multibody systems, nonlinear dynamics, and control, ASME international design engineering technical conferences, San Diego, CA, 30 August-2 September 2009, pp.597-606. 